\title{
Oxygen Reduction Reaction in Conducting Polymer PEDOT: Density Functional Theory Study
}

Sandeep Kumar Singh, Xavier Crispin and Igor Zozoulenko

The self-archived postprint version of this journal article is available at Linköping University Institutional Repository (DiVA):

http:// urn.kb.se/ resolve?urn=urn:nbn:se:liu:diva-139187

N.B.: When citing this work, cite the original publication.

Singh, S. K., Crispin, X., Zozoulenko, I., (2017), Oxygen Reduction Reaction in Conducting Polymer PEDOT: Density Functional Theory Study, The J ournal of Physical Chemistry C, 121(22), 12270-

12277. https:// doi.org/ 10.1021/ acs.jpcc.7b03210

Original publication available at:

https:// doi.org/ 10.1021/ acs.jpcc.7b03210

Copyright: American Chemical Society

http:// pubs.acs.org/ 


\title{
Oxygen Reduction Reaction in Conducting Polymer PEDOT: Density Functional Theory Study
}

\author{
Sandeep Kumar Singh, Xavier Crispin, and Igor V. Zozoulenko* \\ Laboratory of Organic Electronics, ITN, \\ Linköping University, Norrköping SE-60174, Sweden
}

(Dated: May 5, 2017)

\begin{abstract}
An oxygen reduction reaction (ORR) mechanism in conducting polymer PEDOT is studied using the density functional theory. It is demonstrated that pure PEDOT chains posses the catalytic activity, where no platinum catalyst or external dopants are needed to sustain the electrocatalysis. This remarkable property of PEDOT is related to the formation of polaronic states, which leads to the decrease of the HOMO-LUMO gap and thus to the enhancement of the reactivity of the system. It is shown that ORR on PEDOT chains can proceed via two pathways, whether via a four-electron process when the oxygen reacts with protons and is reduced directly into water in four steps (Reaction path I) or via the two-electron process leading to formation of the hydrogen peroxide as an intermediate specimen (Reaction path II). Path I is demonstrated to be energetically preferable. This conclusion also holds for ORR on two $\pi-\pi$ stacked chains and ORR for the case when PEDOT is reduced during the reaction. It is also found that ORR on PEDOT effectively proceeds in the presence of $\mathrm{H}_{3} \mathrm{O}^{+}$, but does not occur in the absence of acidic environment.
\end{abstract}

\section{INTRODUCTION}

Oxygen reduction reaction (ORR) occurs at a cathode of a fuel cell where hydrogen ions that have passed the proton exchange membrane combine with the oxygen and electrons to produce water. ${ }^{1,2}$ ORR can proceed via two pathways, whether through the four-electron process when the oxygen reacts with protons and is reduced directly into water,

$$
\mathrm{O}_{2}+4 \mathrm{H}^{+}+4 e^{-} \rightarrow 2 \mathrm{H}_{2} \mathrm{O}
$$

or via the two-electron process leading to the formation of the hydrogen peroxide as an intermediate specimen,

$$
\mathrm{O}_{2}+2 \mathrm{H}^{+}+2 e^{-} \rightarrow \mathrm{H}_{2} \mathrm{O}_{2} \text {. }
$$

ORR is a slow process representing the limiting factor of operation of fuel cells. In conventional fuel cells containing proton exchange membranes and metallic electrodes ORR requires a catalysts, typically platinum $(\mathrm{Pt}) \cdot{ }^{3-5}$ The high price of platinum drives up the entire cost of the fuel cell and makes a widespread utilization of the fuel cell technology difficult. Because of this, massive efforts have been directed on finding new affordable materials or catalysts capable of at least the same performance suitable for a cost-effective commercial mass production. ${ }^{6}$ In particular, much attention has been paid recently to carbonbased cathode materials such as graphite, graphen, carbon nanotubes, fullerene and related nanomaterials. ${ }^{7-11}$ as well as other materials not requiring Pt catalyst ${ }^{12}$ These materials are shown to exhibit excellent electrocatalytic activity and operational stability required for the ORR. A number of theoretical studies using the density function theory ${ }^{13-25}$ and first-principle molecular dynamics approaches ${ }^{26-30}$ have addressed the mechanisms and pathways of the ORR outlining the role of dopants and the nature of electrocatalytic activity in these materials.
A promising class of novel materials for recyclable power and energy storage devices are conducting polymers, ${ }^{32,46}$ in particular PEDOT (poly $(3,4$ ethylenedioxythiophene). ${ }^{33}$ PEDOT represents one of the most important and most studied materials in organic electronics due to its stability, well-established manufacturing technology and excellent electronic and optical properties. ${ }^{34-36}$ PEDOT-based materials have recently demonstrated record-high values for the combined ionic and electronic conductivity, ${ }^{37}$ which makes them highly interesting for fuel cell applications and for metal-air batteries. ${ }^{38,39}$ In a pioneering study Winther-Jensen et al. demonstrated that a PEDOT electrode offers an ORR electrocatalytic performance similar to that of a conventional Pt-catalyzed one. ${ }^{40}$ This work generated a strong interest to further studies of various aspects of ORR in PEDOT. ${ }^{41-53}$ It should be mentioned that electrocatalytic activity of PEDOT has been questioned and the observed catalysis was attributed to unintentional iron oxide residues on a conducting substrate beneath the polymer. ${ }^{54}$ (Note that a similar controversy arises for metal-free catalysis in carbon materials ${ }^{11}$ ). Further studies however confirmed a strong catalytic activity of PEDOT in $\mathrm{Li}^{-} \mathrm{O}_{2}$ batteries ${ }^{50}$ as well as in metal-free PEDOT electrodes. ${ }^{55}$ Also, it has been demonstrated that ORR pathways depend on the polymerization methods, with electrocatalytic discrepancies being attributed to the presence of different residual iron oxide species. ${ }^{56,57}$

Despite the strong interest to ORR in PEDOT, ${ }^{41-57}$ no fundamental theoretical understanding of its catalytic activity is available at the moment. At the same time, a detailed knowledge of the redox mechanisms, energetics and reaction pathways are in critical demand because without this knowledge a further improvement of the performance of PEDOT-based fuel cells will be difficult. In the present paper we use a quantum-mechanical $a b$ initio 
approach and provide a detailed atomistic insight into reduction steps of oxygen on PEDOT chains. We show that ORR proceeds via four-electron process when oxygen reacts with protons and is reduced directly into water. We demonstrate that PEDOT exhibits intrinsic catalytic activity that does not require noble metal catalysts (as in the case of metallic electrodes) or dopants (as in the case of carbon materials). We attribute this remarkable property of PEDOT to the existence of polaronic states in the band gap that facilitate the catalytic action in the oxidized state.

\section{COMPUTATIONAL METHODS}

The ORR on PEDOT was calculated within the density functional approach using GAUSSIAN (G09). ${ }^{59}$ The geometrical optimizations were performed with the range separated hybrid functional $\mathrm{wB}^{\mathrm{B}} \mathrm{7} \mathrm{XD}^{60}$ level of theory which includes $22 \%$ Hartree Fock (HF) exact exchange at a short-range and $100 \% \mathrm{HF}$ exact exchange at a long range, with the additional Grimmes D2 ${ }^{61}$ dispersion correction. The basis set $6-31 \mathrm{G}(\mathrm{d}, \mathrm{p})$ with polarization functions are employed to optimize various structures. Symmetry constraints were not taken into consideration during geometric optimization. The solvent effect is included by using the polarizable continuum model (PCM) as implemented in the GAUSSIAN package. ${ }^{62}$ The charge distribution on each atom was calculated via the Electrostatic Potential (ESP) method.

In the calculations we used PEDOT chains composed of eight EDOT (3,4-ethylenedioxythiophene) units, see Fig. 1, which is consistent with the length of the PEDOT chains reported in the literature. ${ }^{63}$ (Note that we performed calculations for longer PEDOT chains (up to 20 monomer units), and found that the obtained results are not sensitive to the chain length). On each reaction step $i$ the adsorption energy, $\Delta E_{i}$, was calculated. The adsorption energy is defined as the difference between the energy of the whole system including PEDOT and the absorbed molecules and the sum of the energies of the corresponding isolated systems (i.e. PEDOT and molecules before the reaction). Thus, a negative adsorption energy indicates that the adsorbate molecules are energetically favored for adsorption onto the PEDOT surface.

\section{RESULTS AND DISCUSSION}

\section{A. ORR in PEDOT}

\section{Single PEDOT chain}

We consider ORR on PEDOT in an acidic environment when each reaction step is initiated by introducing one proton $\mathrm{H}^{+}$into the system. We start with a PEDOT chain, an oxygen molecule $\mathrm{O}_{2}$ and $\mathrm{H}^{+}$where the later two subsequently form a complex $\mathrm{OOH}^{+}$, see Fig. 2 (b).

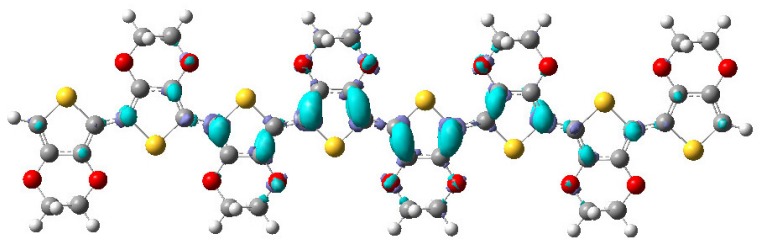

FIG. 1: Calculated Fukui function $f_{N}^{-}(r)$, Eq. (3), shown as colored isodensities. In the PEDOT chain different atoms are indicated as follows: grey (carbon), red (oxygen), yellow (sulfur), small light grey (hydrogen).

In order to find out which atom(s) in the PEDOT chain would be the most efficient in absorbing $\mathrm{OOH}^{+}$we calculate the Fukui function, which predicts the most reactive sites in the structure. ${ }^{64}$ The Fukui function is defined as the change of the electronic density $\rho_{N}(r)$ of the $N$ electron system with respect to the addition/subtraction $(+/-)$ of an electron to/from the system,

$$
f_{N}^{ \pm}(r)= \pm\left(\rho_{N \pm 1}(r)-\rho_{N}(r)\right)
$$

Because we are interested in the reduction reaction when electrons are subtracted from the PEDOT we calculate $f_{N}^{-}(r)$, see Fig. 1. The calculated Fukui function $f_{N}^{-}$has pronounced maximum around $\alpha$ - and $\beta$-carbons, which suggests that these atoms are the most reactive sites in the chain. (For definition of $\alpha$ - and $\beta$-carbons see Fig. $2 \mathrm{a})$.

As mentioned in Introduction, the ORR can proceed via two different reaction pathways including fourelectron four-step process (Path I) or two-electron twostep process (Path II). Below we first summarize the obtained reaction paths, and then proceed with their detailed discussion. 
(a)

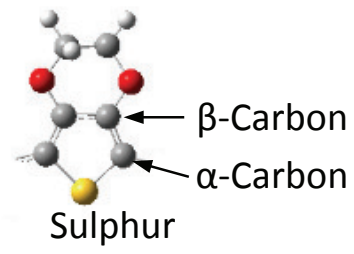

(b)

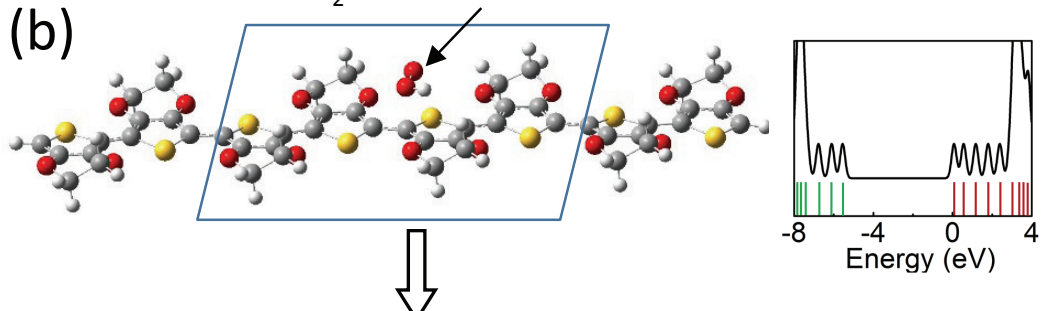

Step 1: PEDOT $+\mathrm{OOH}^{+} \rightarrow \mathrm{PEDOT}^{+}+{ }^{*} \mathrm{OOH}$
Path I

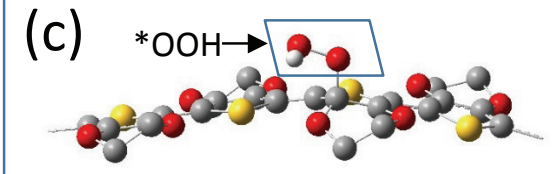

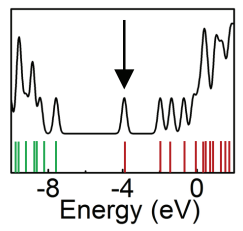

Step 2: $\mathrm{PEDOT}^{+}+{ }^{*} \mathrm{OOH}+\mathrm{H}^{+} \rightarrow \mathrm{PEDOT}^{2+}+{ }^{*} \mathrm{O}+\mathrm{H}_{2} \mathrm{O}$

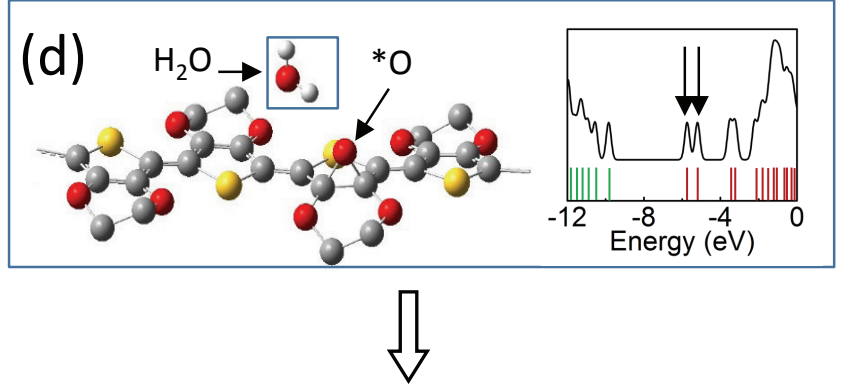

Step 3: PEDOT $^{2+}+{ }^{*} \mathrm{O}+\mathrm{H}_{2} \mathrm{O}+\mathrm{H}^{+} \rightarrow$ PEDOT $^{3+}+* \mathrm{OH}+\mathrm{H}_{2} \mathrm{O}$

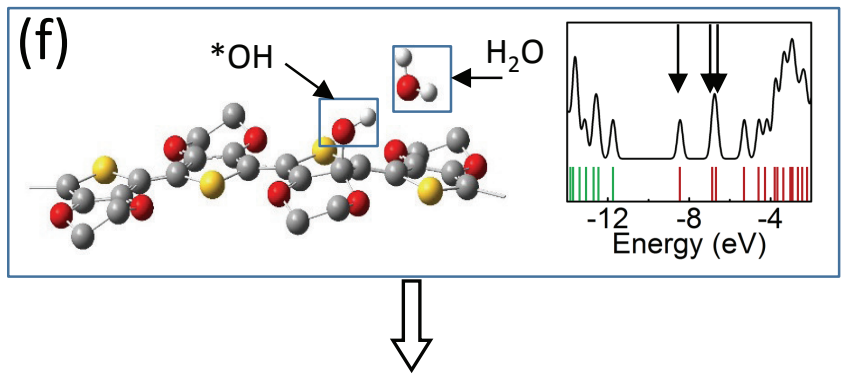

Step 4: PEDOT $^{3+}+{ }^{*} \mathrm{OH}+\mathrm{H}_{2} \mathrm{O}+\mathrm{H}^{+} \rightarrow$ PEDOT $^{4+}+2 \mathrm{H}_{2} \mathrm{O}$

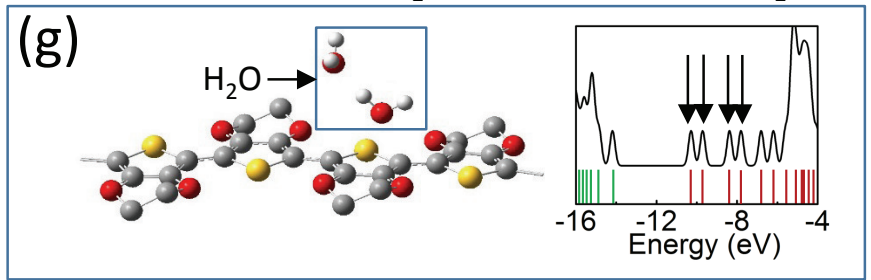

\section{Path II}

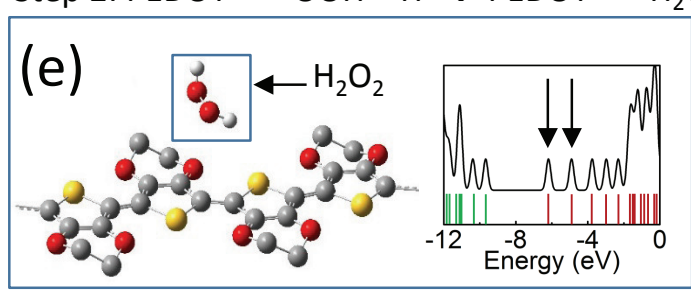

FIG. 2: (a) Definition of absorptions sites of oxygen molecule in a unit cell of PEDOT ( $\alpha$-Carbon, $\beta$-Carbon and Sulphur (S)-atom); the color scheme for PEDOT atoms is the same as in Fig. 1. (c)-(g) correspond to steps 1-4 in the reaction path I, (c),(e) correspond to steps 1-2 in the reaction path II. Insets to the right in (b)-(e) show corresponding energy diagrams including the density of states (black) and the energy levels where polaron states are indicated by arrows. Green and red correspond to the occupied and unoccupied states respectively. 
Reaction path I:

Step 1:

Step 2:

Step 3:

Step 4:

$$
\begin{aligned}
\text { PEDOT }+\mathrm{OOH}^{+} & \rightarrow \mathrm{PEDOT}^{+}+{ }^{*} \mathrm{OOH} \\
\mathrm{PEDOT}^{+}+{ }^{*} \mathrm{OOH}+\mathrm{H}^{+} & \rightarrow \mathrm{PEDOT}^{2+}+{ }^{*} \mathrm{O}+\mathrm{H}_{2} \mathrm{O} \\
\mathrm{PEDOT}^{2+}+{ }^{*} \mathrm{O}+\mathrm{H}_{2} \mathrm{O}+\mathrm{H}^{+} & \rightarrow \mathrm{PEDOT}^{3+}+{ }^{*} \mathrm{OH}+\mathrm{H}_{2} \mathrm{O} \\
\mathrm{PEDOT}^{3+}+{ }^{*} \mathrm{OH}+\mathrm{H}_{2} \mathrm{O}+\mathrm{H}^{+} & \rightarrow \mathrm{PEDOT}^{4+}+2 \mathrm{H}_{2} \mathrm{O}
\end{aligned}
$$

Reaction path II:

Step 1:

$$
\begin{array}{r}
\mathrm{PEDOT}+\mathrm{OOH}^{+} \rightarrow \mathrm{PEDOT}^{+}+{ }^{*} \mathrm{OOH} \\
\mathrm{PEDOT}^{+}+{ }^{*} \mathrm{OOH}+\mathrm{H}^{+} \rightarrow \mathrm{PEDOT}^{2+}+\mathrm{H}_{2} \mathrm{O}_{2},
\end{array}
$$

In the above equations the asterisk * corresponds to atoms/complexes adsorbed on PEDOT.

Step 1 (Reaction paths $I$ and II). The first step of the ORR process for both reaction paths (Eqs. (4),(8)) was simulated by placing $\mathrm{OOH}^{+}$at three different places at a distance of $3 \AA$ from PEDOT chain just above the $\beta$ carbon, $\alpha$-carbon, and the sulfur atom. Let us first focus on the case of $\beta$-carbon, see Fig. 2(b),(c). After geometry optimization we find that $\mathrm{OOH}^{+}$is absorbed on PEDOT with one of its oxygens forming a chemical bond to the $\beta$-carbon atom. The distance between $\beta$-carbon and the oxygen atom reduces to $1.43 \AA$ (corresponding to the $\mathrm{C}-\mathrm{O}$ bond), and the $\beta$-carbon rises out of the plane of PEDOT to form a tetrahedral structure (Fig.2(c)). At the same time, PEDOT donates an electron to ${ }^{*} \mathrm{OOH}^{+}$such that $\beta$-carbon atom becomes positively charged $(\sim+0.6 e)$, see Fig. 3(a). The charge transfer leads to appearance of a positively charged state in the PEDOT which energy lies in the band gap (see the state marked by an arrow in the energy diagram in Fig. 2(c); for a comparison, the energy diagram of a neutral PEDOT (exhibiting no states in the gap) is shown in Fig. 2(b)). The states in the energy gap of PEDOT (as well as of other p-type conducting polymers) represent positively charged localized polarons (or bipolarons) (geometrical distortions of the chains) forming due to the strong electron-lattice coupling between the charge in the chain and the underlying lattice. ${ }^{65,66}$ The molecular orbital corresponding to the polaron state (LUMO) is visualized in Fig. 3(b). The polaronic state is localized on the carbon backbone over 4 units of the chain with the maximum on the units with the absorbed *OOH. For a comparison, the HOMO state is also displayed which exhibits a localization in a different part of the PEDOT chain away from the absorbed * $\mathrm{OOH}$.

In the calculations described above $\mathrm{OOH}^{+}$was placed above the $\beta$-carbon. The placement of $\mathrm{OOH}^{+}$above $\alpha$ carbon or above the sulfur atom shows the same effect, i.e. the absorption of $\mathrm{OOH}^{+}$and charge transfer from PEDOT. The absorption energy of $\mathrm{OOH}^{+}$on different sites ( $\alpha$-carbon, $\beta$-carbon and S-atom) of PEDOT was calculated and it was found to be $\Delta E_{1}=-7.57 \mathrm{eV},-7.54$
$\mathrm{eV}$ and $-5.89 \mathrm{eV}$, respectively, see Fig. 4. This confirms the conclusion based on the Fukui function analysis that $\alpha$-carbon and $\beta$-carbon are the most reactive sites for the ORR reaction. Because the difference between the absorption energies for the cases of $\alpha$-carbon and $\beta$-carbon does not exceed the room temperature thermal energy, for the remaining reaction steps we will limit our analysis to the case of $\beta$-carbon only.

Step 2 (Reaction path I). In step 2, we add $\mathrm{H}^{+}$to the system. Now there are two possibilities for two different reaction paths. For the case of reaction path I (Eq. (5)) $\mathrm{H}^{+}$moves close to the oxygen atom that is bound to $\mathrm{H}$ atom of the absorbed ${ }^{*} \mathrm{OOH}$. Then the electron transfer from PEDOT occurs, the $\mathrm{O}-\mathrm{O}$ bond in ${ }^{*} \mathrm{OOH}$ breaks and a water molecule is formed. At the same time, the remaining $\mathrm{O}$ atom makes a covalent bond with two $\beta$ carbon atoms of PEDOT, see Fig. 2 (d). The absorption energy for this step is $\Delta E_{2}^{I}=-5.23 \mathrm{eV}$, see Fig. 4 (a).

Due to the electron transfer, PEDOT has a total charge of $+2 e$ which leads to a formation a second polaronic state in the gap (both polaronic states are indicated by arrows in the energy diagram in Fig. 2(d)). It should be noted that the electron transfer to ${ }^{*} \mathrm{OOH}$ that has already taken place at step 1 helped to weaken the OO bond whose length became $1.43 \AA$ as compared to $1.32 \AA$ for an isolated $\mathrm{OOH}^{+}$. The bond strength of the $\mathrm{O}-\mathrm{O}$ bond for the isolated $\mathrm{OOH}^{+}$is too high to be broken, thereby limiting the ORR kinetics. The ease of dissociating of the $\mathrm{O}-\mathrm{O}$ bond in the adsorbed ${ }^{*} \mathrm{OOH}$ demonstrates that PEDOT can function as an effective catalyst to facilitate ORR.

Step 2 (Reaction path II). For the case of the reaction path II (Eq. (8)) $\mathrm{H}^{+}$moves to a position near the oxygen atom ${ }^{*} \mathrm{O}$ that is bound to $\beta$-carbon atom after step 1. After the geometry optimization, it was found that $\mathrm{C}-\mathrm{O}$ bond was broken and a hydrogen peroxide molecule $\mathrm{H}_{2} \mathrm{O}_{2}$ was formed. This concludes the reaction path II. The absorption energy for this step is $\Delta E_{2}^{I I}=-3.23 \mathrm{eV}$, see Fig. 4 (a). Because $\left|\Delta E_{2}^{I}\right|>\left|\Delta E_{2}^{I I}\right|$ we conclude that Reaction path I is more thermodynamically favorable than Reaction path II. Note that we also performed this calculation in the presence of water molecules and 
(a) charge distribution

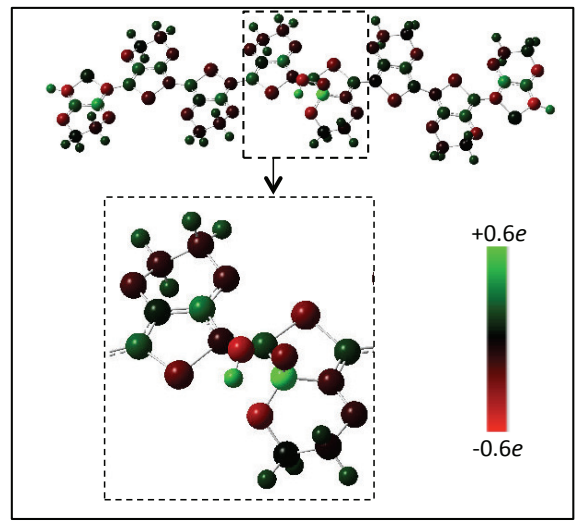

(b) Molecular orbitals

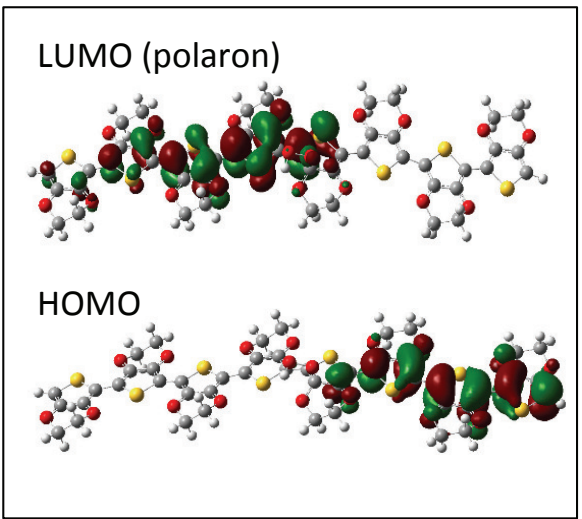

FIG. 3: (a) Charge distribution and (b) molecular orbitals of PEDOT for the reaction step 1. The polaron state (LUMO) is indicated by an arrow in the energy diagram in Fig. 2(b).

found that the reaction product (i.e. $\mathrm{H}_{2} \mathrm{O}_{2}$ ) remains unaffected by water.

Step 3. In step 3 , third $\mathrm{H}^{+}$is added to the system, Eq. (6). It makes a covalent bond to the oxygen and therefore an existing covalent bond between the oxygen and one of $\beta$-carbon atoms gets broken and an absorbed ${ }^{*} \mathrm{OH}$ complex forms (see Fig. 2(f)). An electron transfer takes place again and PEDOT becomes charged with a total charge $+3 e$ accommodating three polaronic states (see a band diagram in Fig. 2(f)). The absorption energy for this step is $\Delta E_{3}^{I}=-3.04 \mathrm{eV}$, Fig. 4 (a).

Step 4. Finally, in step 4 (Eq. (15)), the addition of fourth $\mathrm{H}^{+}$causes breaking the bond between oxygen in ${ }^{*} \mathrm{OH}$ and $\beta$-carbon in PEDOT accompanied by an electron transfer to ${ }^{*} \mathrm{OH}$ and a formation of a covalent bond between $\mathrm{H}^{+}$and $\mathrm{O}$. As a result, a water molecule $\mathrm{H}_{2} \mathrm{O}$ is formed and PEDOT becomes charged with the total charge $+4 e$ accommodating four polaronic states (see a band diagram in Fig. 2(f)). This concludes the ORR reaction on PEDOT for the Reaction path $\mathrm{I}$. The absorption energy for this step is $\Delta E_{4}^{I}=-3.34 \mathrm{eV}$, Fig. 4 (a).

Using the calculated energetics for the four-step ORR reaction we can estimate the work produced by the PEDOT fuel cell. For an each electron transfer step the electrical work is given by the reversible potential $U$ which is related to the change of the Gibbs free energy $\Delta G,{ }^{67,68}$

$$
U=-\frac{\Delta G}{n F}
$$

where $F$ is the Faraday number, and $n$ is the number of electron transferred during a reaction step $(n=1)$. The change of the Gibbs energy $\Delta G$ can be replaced by the reaction energy $E$ as follows, ${ }^{67}$

$$
U=-\frac{E}{n F}-4.6 \mathrm{~V}+c,
$$

where the constant $-4.6 \mathrm{~V}$ accounts for the difference be- tween the electrochemical scale and the vacuum scale used in the quantum-chemical calculations, and the constant $c$ represents the $P V$ and $T S$ energy contributions to the Gibbs free energy. The value of $c$ depends on the reaction models (e.g. environment, solvation, etc), and the details of the density functional theory calculations (exchange-correlation potential, basis sets, etc.), with the reported values of $c$ being in the range $0.5 \mathrm{~V}<c<0.76$ $\mathrm{V}{ }^{67,69}$ The calculated total reaction energy for all electron transfer steps is $E_{t o t}=-19.18 \mathrm{eV}$ (see Fig. 4). Summing up the reversible potentials, Eq. (11), for all four reactions steps, using $c \approx 0.6 \mathrm{~V}$ and substituting there the calculated value of $E_{t o t}$ we obtain the work done by the fuel cell $e U \approx 3.18 \mathrm{eV}$. This value is somehow lower than the corresponding theoretical value for the four-step process $4 \times 1.23 \mathrm{eV}=4.92 \mathrm{eV}$, where $U_{0}=1.23$ $\mathrm{V}$ is the standard reversible potential on the standard hydrogen electrode scale. ${ }^{68}$

\section{Reducing PEDOT during ORR}

During ORR positive charges are accumulated on the PEDOT chains and at the last step of the reaction each PEDOT chain contains four positive charges, Eq. (15). To keep a fuel cell operating and to maintain a current flow in the circuit these charges have to be removed from the cathode, which is achieved by application of a negative potential to the PEDOT electrode. As a result, PEDOT is continuously reduced towards its neutral state. In the present section we investigate how the reduction of PEDOT affects the efficiency of ORR on a PEDOT chain. To this end, at each step of ORR we reduce PEDOT (i.e. add electrons to the PEDOT chain). Calculations show that in this case ORR proceeds exactly through the same steps as in the case discussed in Sec. III A 1. That is, the reaction steps read, 


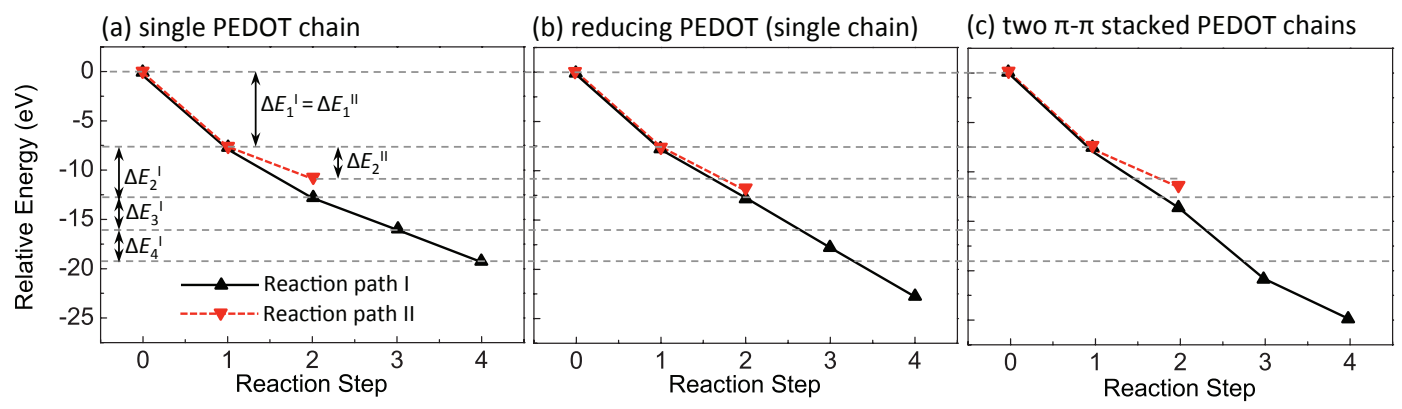

FIG. 4: Relative energies for the reaction pathways of ORR (a) for a single PEDOT chain; (b) for the case of reduction of PEDOT during ORR; (c) for two $\pi-\pi$ stacked PEDOT chains. The definition of absorption energies $\Delta E_{i}^{I(I I)}$ is visualized in (a) where $i=1-4$ correspond to the reaction steps, and I(II) corresponds to the reaction path I(II). Horizontal dashed lines are guides for eye.

Reaction path I:

Step 1:

$\mathrm{PEDOT}+\mathrm{OOH}^{+} \rightarrow \mathrm{PEDOT}^{+}+{ }^{*} \mathrm{OOH}$

Step 2:

$\mathrm{PEDOT}^{+}+e \rightarrow$ PEDOT

Step 3:

$\mathrm{PEDOT}+{ }^{*} \mathrm{OOH}+\mathrm{H}^{+} \rightarrow \mathrm{PEDOT}^{+}+{ }^{*} \mathrm{O}+\mathrm{H}_{2} \mathrm{O}$

$\mathrm{PEDOT}^{+}+e \rightarrow$ PEDOT

PEDOT $+{ }^{*} \mathrm{O}+\mathrm{H}_{2} \mathrm{O}+\mathrm{H}^{+} \rightarrow \mathrm{PEDOT}^{+}+{ }^{*} \mathrm{OH}+\mathrm{H}_{2} \mathrm{O}$

$\mathrm{PEDOT}^{+}+e \rightarrow$ PEDOT

Step 4:

PEDOT $+{ }^{*} \mathrm{OH}+\mathrm{H}_{2} \mathrm{O}+\mathrm{H}^{+} \rightarrow \mathrm{PEDOT}^{+}+2 \mathrm{H}_{2} \mathrm{O}$

$\mathrm{PEDOT}^{+}+e \rightarrow \mathrm{PEDOT}$

Reaction path II:

Step 1:

$\mathrm{PEDOT}+\mathrm{OOH}^{+} \rightarrow \mathrm{PEDOT}^{+}+{ }^{*} \mathrm{OOH}$

Step 2:

$\mathrm{PEDOT}^{+}+e \rightarrow \mathrm{PEDOT}$

$\mathrm{PEDOT}^{+}+{ }^{*} \mathrm{OOH}+\mathrm{H}^{+} \rightarrow \mathrm{PEDOT}^{2+}+\mathrm{H}_{2} \mathrm{O}_{2}$,

$\mathrm{PEDOT}^{+}+e \rightarrow$ PEDOT

The calculated absorption energies for steps 1-4 are respectively $\Delta E_{1}^{I}=\Delta E_{1}^{I I}=-7.53 \mathrm{eV}, \Delta E_{2}^{I}=-4.95$ $\mathrm{eV}, \Delta E_{3}^{I}=-5.18 \mathrm{eV}$, and $\Delta E_{4}^{I}=-4.97 \mathrm{eV}$, see Fig. 4 (b). The calculated absorption energies $\Delta E_{i}^{I}$ are somehow larger than corresponding energies calculated without PEDOT reduction (except step 2), which means that for the case of the reduced PEDOT the ORR can progress even more efficiently than for the oxidized one. For the case of Reaction path II the calculated absorption energy for the second step is $\Delta E_{2}^{I I}=-4.43 \mathrm{eV}$, which is smaller than the corresponding value for path I ( -4.95 $\mathrm{eV})$. This means that for the case of reduced PEDOT Reaction path I is also more efficient than Reaction path II.

\section{ORR on two $\pi-\pi$ stacked PEDOT chains}

A PEDOT film is largely amorphous with a limited crystallic order, with PEDOT chains being assembled in small $\pi-\pi$ stacked crystallites typically consisting of 3-10 chains. ${ }^{66,70-72}$ It is therefore important to investigate how a $\pi$ - $\pi$ stacking affects the ORR reaction. We proceed in the same way as in Sec. III A 1 placing $\mathrm{OOH}^{+}$above the $\beta$-carbon of the upper PEDOT chain, see Fig. 5. After geometry optimization we find that $\mathrm{OOH}^{+}$is absorbed on PEDOT with one of its oxygens forming a chemical bond to the $\beta$-carbon atom. The calculated geometry of the absorbed * $\mathrm{OOH}$ on two $\pi-\pi$ stacked chains is practically the same as the one for the single chain, cf. Fig. 2 (c) and Fig. 5. Subsequent additions of $\mathrm{H}^{+}$to the system shows that for the case of two $\pi-\pi$ stacked chains ORR proceeds exactly through the same steps as for the single chain 


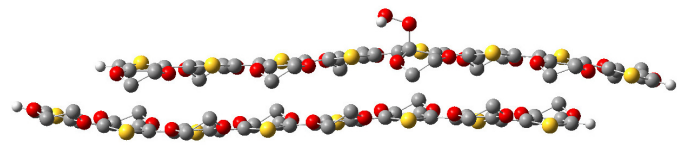

FIG. 5: Optimized structure of two $\pi-\pi$ PEDOT stacked PEDOT after the first reaction step. Oxygen molecule in absorbed ${ }^{*} \mathrm{OOH}^{+}$is covalently bound to $\beta$-carbon, cf. Fig. 2(a) showing step 1 for a single PEDOT chain.

discussed in Sec. III A 1, Eqs. (4)-(9). The calculated absorption energies for steps 1-4 are respectively $\Delta E_{1}^{I}=$ $\Delta E_{1}^{I I}=-7.81 \mathrm{eV}, \Delta E_{2}^{I}=-5.93 \mathrm{eV}, \Delta E_{3}^{I}=-7.21 \mathrm{eV}$, and $\Delta E_{4}^{I}=-3.95 \mathrm{eV}$ and for the second step of Reaction path II, $\Delta E_{2}^{I I}=-3.78 \mathrm{eV}$, see Fig. 4 (c). As for the case of a single chain, Reaction path I on two $\pi-\pi$ stacked chains is also more effective than Reaction path II.

\section{B. Mechanism of the catalytic action of PEDOT}

The results presented in the previous sections show that pure PEDOT chains posses the catalytic property (i.e. they do not require any external dopants to facilitate the reaction). This is in strong contrast to the case of metallic electrodes which require a platinum catalyst or to the case of graphene where doped graphene have catalytic capability but pure graphene does not. We relate this remarkable property of PEDOT to a formation of polaronic states in the gap during the reaction (see energy diagrams in Fig. 2 and a related discussion in Sec. III A 1). Indeed, the energy difference between LUMO and HOMO represents an indicator of chemical reactivity; the smaller this difference is, the faster the reaction proceeds. ${ }^{58,74}$ At each reaction step, due to an easily donating nature of PEDOT (which is a p-type material), a charge transfer occurs, which results in the creation of a positive polaronic state in the chain, see Fig. 3. This state represents a new LUMO state whose energy is much lower than the LUMO energy of a corresponding neutral chain. Thus, the HOMO-LUMO gap is significantly reduced, which eventually leads of the enhancement of the reactivity of PEDOT. It is noteworthy that polaronic states are localized on the PEDOT chain (i.e. not at the absorbed species), which enables accepting positive charges and thus efficient catalytic action during ORR.

Note that conceptually similar explanation of the catalytic capability applies to the doped graphene where the HOMO-LUMO gap is strongly reduced after a dopant atom is substituted into the graphene sheet thus creating a state in the gap. ${ }^{14}$

\section{Factors affecting ORR reaction in PEDOT}

In the previous section we outlined the basics of ORR on PEDOT discussing reactions paths, steps, and reac-

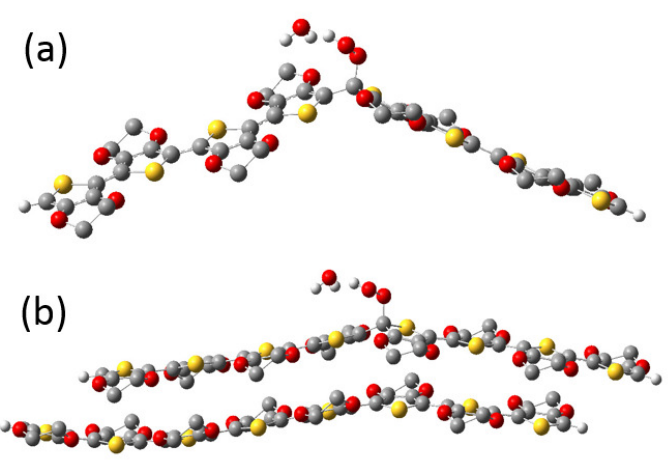

FIG. 6: Optimized structure of (a) a PEDOT chain and (b) two $\pi-\pi$ stacked PEDOT chains (b) in the presence of an oxygen molecule and $\mathrm{H}_{3} \mathrm{O}^{+}$during step 1 . Oxygen molecule is covalently bound to $\alpha$-carbon.

tion energetics. In the present section we consider the effect of various factors that might influence the ORR in PEDOT.

\section{ORRs on PEDOT and $\pi-\pi$ stacked PEDOT in the presence of $\mathrm{H}_{3} \mathrm{O}^{+}$}

Because of the presence of water and protons in the cathode electrode one can expect that some water molecules get protonated forming hydronium, $\mathrm{H}_{3} \mathrm{O}^{+}$. It is therefore of interest to analyze the ORR process on PEDOT and $\pi-\pi$ stacked PEDOT in the presence of $\mathrm{H}_{3} \mathrm{O}^{+}$. We start calculations by placing $\mathrm{H}_{3} \mathrm{O}^{+}$and $\mathrm{O}_{2}$ above a PEDOT chain. During optimization process, the $\mathrm{H}^{+}$de-attaches from $\mathrm{H}_{3} \mathrm{O}^{+}$and makes covalent bond to one of atoms of the oxygen molecule, $\mathrm{H}_{3} \mathrm{O}^{+}+\mathrm{O}_{2} \rightarrow$ $\mathrm{H}_{2} \mathrm{O}+\mathrm{OOH}^{+}$. Then the other oxygen atom of $\mathrm{OOH}^{+}$ complex makes a covalent bond to the $\alpha$-carbon of the PEDOT chain, PEDOT $+\mathrm{OOH}^{+} \rightarrow \mathrm{PEDOT}^{+}+{ }^{*} \mathrm{OOH}$. This is accompanied by an electron transfer to the absorbed ${ }^{*} \mathrm{OOH}$ and thus an appearance of a polaron state on PEDOT, see Fig. 6. This corresponds to Step 1 described in previous sections, Eq (4), which means that $\mathrm{H}_{3} \mathrm{O}^{+}$effectively mediates ORR on PEDOT.

\section{Absence of acidic environment}

In this section we investigate the catalytic behavior of PEDOT in the absence of acidic environment. We place oxygen molecules at different positions in the vicinity of PEDOT chain when no protons are present in the systems. We consider cases with and without water. After the geometry optimization we find no evidence of catalytic activity of PEDOT, see Fig. 7. This is in an agreement with experimental electrochemical investigations of Khomenko et al..$^{75}$ who studied PEDOT catalytic electrodes in oxygen-saturated electrolytes and found no 


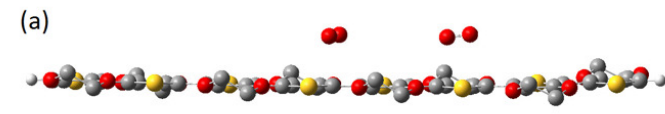

(b)

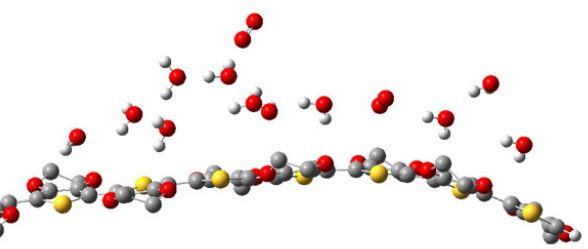

FIG. 7: Optimized PEDOT in the presence of oxygen molecules (a) without water and (b) with water. Oxygen molecules do not make covalent bonds with PEDOT in both cases.

evidence of the electrocatalytic activity towards the oxygen reduction. The absence of the catalytic activity is fully consistent with the mechanism of the catalytic action of PEDOT outlined in Sec. IIIB. Indeed, in the absence of protons, the electron transfer from PEDOT does not take place. As a result, no polaronic state in the gap facilitating ORR is formed.

\section{CONCLUSION}

A conducting polymer PEDOT represents a promising material for recyclable power and energy storage devices including fuel cells. A number of experimental works addressed the oxygen reduction reaction (ORR) on the PEDOT electrode, which is critical for fuel cell applications. In the present study we, for the first time to the best of our knowledge, report theoretical studies of the ORR on PEDOT. Using quantum-mechanical calculations based on the density functional theory we demonstrate that in the acidic environment ORR on a PEDOT chain can proceed via two pathways, whether via the four-electron process when the oxygen reacts with protons and is reduced directly into water in four steps (Reaction path I) or via the two-electron process leading to formation of the hydrogen peroxide as an intermediate specimen (Reaction path II). We optimized the geometrical structure and calculated molecular orbitals and charge distribution in PEDOT chains. We studied the energetics of the reaction and found that the energy of the system decreases for each reaction step, which indicates that ORR occurs spontaneously on PEDOT. The calculated absorption energy shows that the decrease in the ground state energy for reaction path I is larger than that of reaction path II, and therefore we conclude that ORR on PEDOT favors the Reaction path I. Using the Fukui function analysis we identify the reactive sites of the reaction. We also consider ORR on two $\pi-\pi$ stacked PEDOT chains and ORR for the case when PEDOT is reduced during the reaction, and in both cases we find that Reaction path I is more energetically favorable.

Our results demonstrate that pure PEDOT chains posses the catalytic property (i.e. they do not require any external dopants to facilitate the reaction). This is in strong contrast to the case of metallic electrodes which require a platinum catalyst or to the case of graphene where doped graphene have catalytic capability but pure graphene does not. We relate this remarkable property of PEDOT to a formation of polaronic states, which leads to the reduction of the HOMO-LUMO gap and thus to the enhancement of the reactivity of the system.

We also investigate the effect of various factors that might influence the ORR in PEDOT. In particular, we find that ORR on PEDOT can effectively proceed in the presence of $\mathrm{H}_{3} \mathrm{O}^{+}$, but does not occur in the absence of acidic environment.

\section{ACKNOWLEDGMENTS}

This work was supported by the Swedish Energy Agency (38332-1), the Knut and Alice Wallenberg Foundation through the project The Tail of the Sun, Carl Trygges foundation (CTS: 13 527), Troëdssons Research Foundation (896/16), and J. Gust. Richert Foundation (2016-00242). We acknowledge the support from the Swedish Research Council via "Research Environment grant" on "Disposable paper fuel cells" (2016-05990). The computations were performed on resources provided by the Swedish National Infrastructure for Computing (SNIC) at NSC.
* Electronic address: igor.zozoulenko@liu.se

1 Haile, S. M. Fuel Cell Materials and Components. Acta Mater. 2003, 51, 5981-6000.

2 Litster, S.; McLean, G. Pem Fuel Cell Electrodes. J. Power Sources 2004, 130, 61-76.

3 Stephens, I. E. L.; Bondarenko, A. S.; Gronbjerg, U.; Rossmeisl, J.; Chorkendorff, I. Understanding the Electrocatalysis of Oxygen Reduction on Platinum and It's Alloys. Energy Environ. Sci. 2012, 5, 6744-6762.

4 Nørskov, J. K.; Rossmeisl, J. K.; Logadottir, A; Lindqvist,
L; Kitchin, J. R.; Bligaard, T; Jónsson, H. Origin of the Overpotential for Oxygen Reduction at a Fuel-Cell Cathode. J. Phys. Chem. 2004, 108, 17886-17892.

5 Karlberg, G. S.; Rossmeisl, J.; Nørskov J. K. Estimations of electric field effects on the oxygen reduction reaction based on the density functional theory. Phys. Chem. Chem. Phys. 2007, 9, 5158-5161.

6 Sealy, C. The Problem with Platinum. Mater. Today 2008, 11, 65-68.

7 Dai, L.; Xue, Y.; Qu, L.; Choi, H.-J.; Baek, J.-B. Metal- 
Free Catalysts for Oxygen Reduction Reaction. Chem. Rev. 2015, 115, 4823-4892.

${ }^{8} \mathrm{Hu}$, C.; Dai, L. Carbon-Based Metal-Free Catalysts for Electrocatalysis beyond the ORR. Angew. Chem., Int. Ed. 2016, 55, 11736-11758.

9 Shao, M.; Chang, Q.; Dodelet, J.-P.; Chenitz, R. Recent Advances in Electrocatalysts for Oxygen Reduction Reaction. Chemical Reviews 2016, 116, 3594-3657.

10 Wu, K.-H.; Wang, D.-W.; Su, D.-S.; Gentle, I. R. A Discussion on the Activity Origin in Metal-Free Nitrogen-Doped Carbons for Oxygen Reduction Reaction and Their Mechanisms. ChemSusChem 2015, 8, 2772-2788.

11 Masa, J.; Xia, W.; Muhler, M.; Schuhmann, W. On the Role of Metals in Nitrogen-Doped Carbon Electrocatalysts for Oxygen Reduction. Angew. Chem., Int. Ed. 2015, 54, 10102-10120.

12 Sun, S.; Jiang, N.; Xia, D. Density Functional Theory Study of the Oxygen Reduction Reaction on Metalloporphyrins and Metallophthalocyanines. J. Phys. Chem. C 2011, 115, 9511-9517.

13 Sidik, R. A.; Anderson, A. B.; Subramanian, N. P.; Kumaraguru, S. P.; Popov, B. N. $\mathrm{O}_{2}$ Reduction on Graphite and Nitrogen-Doped Graphite: Experiment and Theory. J. Phys. Chem. B 2006, 110, 1787-1793.

14 Zhang, L.; Xia, Z. Mechanisms of Oxygen Reduction Reaction on Nitrogen-Doped Graphene for Fuel Cells. J. Phys. Chem. C 2011, 115, 11170-11176.

${ }^{15}$ Kim, H.; Lee, K.; Woo, S. I.; Jung, Y. On the Mechanism of Enhanced Oxygen Reduction Reaction in NitrogenDoped Graphene Nanoribbons. Phys. Chem. Chem. Phys. 2011, 13, 17505-17510.

16 Yu, L.; Pan, X.; Cao, X.; Hu, P.; Bao, X. Oxygen Reduction Reaction Mechanism on Nitrogen-Doped Graphene: A Density Functional Theory Study. J. Catal. 2011, 282, 183-190.

17 Boukhvalov, D. W.; Son, Y.-W. Oxygen Reduction Reactions on Pure and Nitrogen-Doped Graphene: A FirstPrinciples Modeling. Nanoscale 2012, 4, 417-420.

18 Zhang, L.; Niu, J.; Dai, L.; Xia, Z. Effect of Microstructure of Nitrogen-Doped Graphene on Oxygen Reduction Activity in Fuel Cells. Langmuir 2012, 28, 7542-7550.

19 Wu, P.; Du, P.; Zhang, H.; Cai, C. Graphyne as a Promising Metal-Free Electrocatalyst for Oxygen Reduction Reactions in Acidic Fuel Cells: A DFT Study. J. Phys. Chem. $C$ 2012, 116, 20472-20479.

20 Yang, L.; Jiang, S.; Zhao, Y.; Zhu, L.; Chen, S.; Wang, X.; Wu, Q.; Ma, J.; Ma, Y.; Hu, Z. Boron-Doped Carbon Nanotubes as Metal-Free Electrocatalysts for the Oxygen Reduction Reaction. Angew. Chem., Int. Ed. 2011, 50, 7132-7135.

${ }^{21}$ Fazio, G.; Ferrighi, L.; Perilli, D.; Di Valentin, C. Computational Electrochemistry of Doped Graphene as Electrocatalytic Material in Fuel Cells. Int. J. Quantum Chem. 2016, 116, 1623-1640.

22 Zhao, Z.; Li, M.; Zhang, L.; Dai, L.; Xia, Z. Design Principles for Heteroatom-Doped Carbon Nanomaterials as Highly Efficient Catalysts for Fuel Cells and Metal-Air Batteries. Adv. Mater. 2015, 27, 6834-6840.

${ }^{23}$ Liang, W.; Chen, J.; Liu, Y.; Chen, S. Density-FunctionalTheory Calculation Analysis of Active Sites for FourElectron Reduction of $\mathrm{O}_{2}$ on Fe/N-Doped Graphene. ACS Catal. 2014, 4, 4170-4177.

${ }^{24} \mathrm{Li}$, M.; Zhang, L.; Xu, Q.; Niu, J.; Xia, Z. N-Doped Graphene as Catalysts for Oxygen Reduction and Oxy- gen Evolution Reactions: Theoretical Considerations. J. Catal. 2014, 314, 66-72.

${ }^{25}$ Hughes, Z. E.; Walsh, T. R. Computational Chemistry for Graphene-Based Energy Applications: Progress and Challenges. Nanoscale 2015, 7, 6883-6908.

${ }^{26}$ Ikeda, T.; Boero, M.; Huang, S.-F.; Terakura, K.; Oshima, M.; Ozaki, J.-I. Carbon Alloy Catalysts: Active Sites for Oxygen Reduction Reaction. J. Phys. Chem. C 2008, 112, 14706-14709.

27 Okamoto, Y. First-Principles Molecular Dynamics Simulation of $\mathrm{O}_{2}$ Reduction on Nitrogen-Doped Carbon. Appl. Surf. Sci. 2009, 256, 335-341.

28 Ikeda, T.; Boero, M.; Huang, S.-F.; Terakura, K.; Oshima, M.; Ozaki, J.-I.; Miyata, S. Enhanced Catalytic Activity of Carbon Alloy Catalysts Codoped with Boron and Nitrogen for Oxygen Reduction Reaction. J. Phys. Chem. C 2010, 114, 8933-8937.

${ }^{29}$ Koizumi, K.; Nobusada, K.; Boero, M. Reaction Pathway and Free Energy Landscape of Catalytic Oxidation of Carbon Monoxide Operated by a Novel Supported Gold-Copper Alloy Cluster. J. Phys. Chem. C 2015, 119, 15421-15427.

${ }^{30}$ Chai, G.-L.; Hou, Z.; Shu, D.-J.; Ikeda, T.; Terakura, K. Active Sites and Mechanisms for Oxygen Reduction Reaction on Nitrogen-Doped Carbon Alloy Catalysts: StoneWales Defect and Curvature Effect. J. Am. Chem. Soc. 2014, 136, 13629-13640.

31 Abdelhamid, M. E.; O'Mullane, A. P.; Snook, G. A. Storing Energy in Plastics: A Review on Conducting Polymers \& Their Role in Electrochemical Energy Storage. RSC Adv. 2015, 5, 11611-11626.

32 Zhou, Q.; Shi, G. Conducting Polymer-Based Catalysts. J. Am. Chem. Soc. 2016, 138, 2868-2876.

${ }^{33}$ Sun, K.; Zhang, S.; Li, P.; Xia, Y.; Zhang, X.; Du, D.; Isikgor, F. H.; Ouyang, J. Review on Application of Pedots and Pedot:Pss in Energy Conversion and Storage Devices. J. Mater. Sci.: Mater. Electron. 2015, 26, 4438-4462.

34 Lövenich, W. Pedot-Properties and Applications. Polym. Sci. Ser. C 2014, 56, 135-143.

35 Elschner, A.; Kirchmeyer, S.; Lovenich, W.; Merker, U.; Reuter, K. Pedot : Principles and Applications of an Intrinsically Conductive Polymer; CRC Press: Boca Raton, FL, 2011.

36 Shi, H.; Liu, C.; Jiang, Q.; Xu, J. Effective Approaches to Improve the Electrical Conductivity of Pedot:Pss: A Review. Adv. Electron. Mater. 2015, 1, 1500017.

37 Malti, A.; Edberg, J.; Granberg, H.; Khan, Z. U.; Andreasen, J. W.; Liu, X.; Zhao, D.; Zhang, H.; Yao, Y.; Brill, J. W.; et al. An Organic Mixed Ion-Electron Conductor for Power Electronics. Adv. Sci. 2016, 3, 1500305.

38 Cheng, F.; Chen, J. Metal-Air Batteries: From Oxygen Reduction Electrochemistry to Cathode Catalysts. Chem. Soc. Rev. 2012, 41, 2172-2192.

39 Cao, R.; Lee, J.-S.; Liu, M.; Cho, J. Recent Progress in Non-Precious Catalysts for Metal-Air Batteries. Adv. Energy Mater. 2012, 2, 816-829.

40 Winther-Jensen, B.; Winther-Jensen, O.; Forsyth, M.; MacFarlane, D. R. High Rates of Oxygen Reduction over a Vapor Phase-Polymerized Pedot Electrode. Science 2008, 321, 671-674.

41 Amanchukwu, C. V.; Gauthier, M.; Batcho, T. P.; Symister, C.; Shao-Horn, Y.; D'Arcy, J. M.; Hammond, P. T. Evaluation and Stability of Pedot Polymer Electrodes for Li-O ${ }_{2}$ Batteries. J. Phys. Chem. Lett. 2016, 7, 3770-3775. 
42 Bayatsarmadi, B.; Peters, A.; Talemi, P. Catalytic Polymeric Electrodes for Direct Borohydride Fuel Cells. J. Power Sources 2016, 322, 26-30.

43 Vigil, J. A.; Lambert, T. N.; Eldred, K. Electrodeposited $\mathrm{MnO}_{x} /$ Pedot Composite Thin Films for the Oxygen Reduction Reaction. ACS Appl. Mater. Interfaces 2015, 7, 22745-22750.

${ }^{44}$ Kuo, Y.-L.; Wu, C.-C.; Chang, W.-S.; Yang, C.-R.; Chou, H.-L. Study of Poly (3,4-Ethylenedioxythiophene)/ $\mathrm{MnO}_{2}$ as Composite Cathode Materials for Aluminum-Air Battery. Electrochim. Acta 2015, 176, 1324-1331.

45 Webb, H. K.; Notley, S. M.; Evans, D. R. Observation of Electron Transfer between Bacteria and High Conductivity Graphene-Pedot Composites. RSC Adv. 2015, 5, 45642-45645.

46 Abdelhamid, M. E.; Snook, G. A.; O’Mullane, A. P. Electropolymerisation of Catalytically Active Pedot from an Ionic Liquid on a Flexible Carbon Cloth Using a Sandwich Cell Configuration. ChemPlusChem 2015, 80, 74-82.

47 Guo, Z.; Liu, H.; Jiang, C.; Zhu, Y.; Wan, M.; Dai, L.; Jiang, L. Biomolecule-Doped Pedot with ThreeDimensional Nanostructures as Efficient Catalyst for Oxygen Reduction Reaction. Small 2014, 10, 2087-2095.

48 Zhang, M.; Yuan, W.; Yao, B.; Li, C.; Shi, G. SolutionProcessed Pedot:Pss/Graphene Composites as the Electrocatalyst for Oxygen Reduction Reaction. ACS Appl. Mater. Interfaces 2014, 6, 3587-3593.

${ }^{49}$ Gustafson, M. P.; Matsumoto, K.; MacFarlane, D. R.; Winther-Jensen, B. An Investigation of the Properties of Conducting Polymer Alloys for Water Oxidation. Electrochim. Acta 2014, 122, 166-172.

${ }^{50}$ Nasybulin, E.; Xu, W.; Engelhard, M. H.; Li, X. S.; Gu, M.; Hu, D.; Zhang, J.-G. Electrocatalytic Properties of Poly(3,4-Ethylenedioxythiophene) (Pedot) in $\mathrm{Li}_{2} \mathrm{O}_{2}$ Battery. Electrochem. Commun. 2013, 29, 63-66.

${ }^{51}$ Latonen, R.-M.; Wang, X.; Sjöberg-Eerola, P.; Eriksson, J.-E.; Bergelin, M.; Bobacka, J. Poly(3,4Ethylenedioxythiophene) Based Enzyme-Electrode Configuration for Enhanced Direct Electron Transfer Type Biocatalysis of Oxygen Reduction. Electrochim. Acta 2012, $68,25-31$

${ }^{52}$ Xuan, Y.; Sandberg, M.; Berggren, M.; Crispin, X. An All-Polymer-Air Pedot Battery. Org. Electron. 2012, 13, 632-637.

53 Winther-Jensen, B.; Fraser, K.; Ong, C.; Forsyth, M.; MacFarlane, D. R. Conducting Polymer Composite Materials for Hydrogen Generation. Adv. Mater. 2010, 22, $1727-1730$.

${ }^{54}$ Gu, C.; Norris, B. C.; Fan, F.-R. F.; Bielawski, C. W.; Bard, A. J. Is Base-Inhibited Vapor Phase Polymerized Pedot an Electrocatalyst for the Hydrogen Evolution Reaction? Exploring Substrate Effects, Including Pt Contaminated Au. ACS Catal. 2012, 2, 746-750.

55 Cottis, P. P.; Evans, D.; Fabretto, M.; Pering, S.; Murphy, P.; Hojati-Talemi, P. Metal-Free Oxygen Reduction Electrodes Based on Thin Pedot Films with High Electrocatalytic Activity. RSC Adv. 2014, 4, 9819-9824.

56 Kerr, R.; Pozo-Gonzalo, C.; Forsyth, M.; Winther-Jensen, B. Influence of the Polymerization Method on the Oxygen Reduction Reaction Pathway on Pedot. ECS Electrochem. Lett. 2013, 2, F29-F31.

57 Kerr, R.; Pozo-Gonzalo, C.; Forsyth, M.; Winther-Jensen, B. The Reduction of Oxygen on Iron(Ii) Oxide/Poly(3,4Ethylenedioxythiophene) Composite Thin Film Elec- trodes. Electrochim. Acta 2015, 154, 142-148.

58 Zhou, Z.; Parr, R. G. Activation Hardness: New Index for Describing the Orientation of Electrophilic Aromatic Substitution. J. Am. Chem. Soc. 1990, 112, 5720-5724.

59 Frisch, M. J.; Trucks, G. W.; Schlegel, H. B.; Scuseria, G. E.; Robb, M. A.; Cheeseman, J. R.; Scalmani, G.; Barone, V.; Mennucci, B.; Petersson, G. A., et al. Gaussian 09, Revision E.1; Gaussian, Inc.: Wallingford, CT, 2009.

${ }^{60}$ Chai, J.-D.; Head-Gordon, M. Long-Range Corrected Hybrid Density Functionals with Damped Atom-Atom Dispersion Corrections. Phys. Chem. Chem. Phys. 2008, 10, 6615-6620.

${ }^{61}$ Grimme, S. Semiempirical GGA-Type Density Functional Constructed with a Long- Range Dispersion Correction. J. Comput. Chem. 2010, 27, 1787-1799.

${ }^{62}$ Tomasi, J.; Mennucci, B.; Cammi, R. Quantum Mechanical Continuum Solvation Models. Chem. Rev. 2005105 , 2999-3093.

${ }^{63}$ Ugur, A.; Katmis, F.; Li, M.; Wu, L.; Zhu, Y.; Varanasi, K. K.; Gleason, K. K. Low-Dimensional Conduction Mechanisms in Highly Conductive and Transparent Conjugated Polymers. Adv. Mater. 2015, 27, 4604-4610.

${ }^{64}$ Parr, R. G.; Yang, W. Density Functional Approach to the Frontier-Electron Theory of Chemical Reactivity. J. Am. Chem. Soc. 1984, 106, 4049-4050.

${ }^{65}$ Salaneck, W. R.; Friend, R. H.; Brédas, J. L. Electronic Structure of Conjugated Polymers: Consequences of Electron-Lattice Coupling. Phys. Rep. 1999, 319, 231-251.

66 Muñoz, W. A.; Singh, S. K.; Franco-Gonzalez J. F.; Linares, M.; Crispin, X.; Zozoulenko, I. V. Insulator to Semimetallic Transition in Conducting Polymers. Phys. Rev. B 2016, 94, 205202.

67 Roques, J; Anderson, A. B.; $\mathrm{Pt}_{3} \mathrm{Cr}(111)$ Alloy Effect on the Reversible Potential of $\mathrm{OOH}(\mathrm{ads})$. Formation from $\mathrm{O}_{2}$ (ads) relative to $\mathrm{Pt}(111)$ J. Fuel Cell Sci. Technol. 2005, $2,86-93$.

68 Anderson, A. B.; Insights into Electrocatalysis. Phys. Chem. Chem. Phys. 2012, 14, 1330-1338.

69 Anderson, A. B.; Theory at the electrochemical interface: reversible potentials and potential-dependent activation energies. Electrochim. Acta 2003, 48, 3743??-3749.

70 Takano, T.; Masunaga, H.; Fujiwara, A.; Okuzaki, H.; Sasaki, T. PEDOT Nanocrystal in Highly Conductive PEDOT:PSS Polymer Films. Macromolecules 2012, 45, 3859-3865.

71 Palumbiny, C. M.; Schlipf, J.; Hexemer, A.; Wang, C.; Müller-Buschbaum, P. The Morphological Power of Soap: How Surfactants Lower the Sheet Resistance of PEDOT:PSS by Strong Impact on Inner Film Structure and Molecular Interface Orientation. Adv. Electron. Mater. 2016, 2, 1500377.

72 Franco-Gonzalez J. F.; Zozoulenko, I. V. Molecular Dynamics Study of Morphology of Doped PEDOT: From Solution to Dry Phase. J. Phys. Chem. B 2017, 121, 4299-4307.

73 Wang, Y.; Balbuena, P. B. Ab Initio Molecular Dynamics Simulations of the Oxygen Reduction Reaction on a $\mathrm{Pt}(111)$ Surface in the Presence of Hydrated Hydronium $\left(\mathrm{H}_{3} \mathrm{O}\right)^{+}\left(\mathrm{H}_{2} \mathrm{O}\right)_{2}$ : Direct or Series Pathway? J. Phys. Chem. $B$ 2005, 109, 14896-14907.

74 Aihara, J. -I. Reduced HOMO-LUMO Gap as an Index of Kinetic Stability for Polycyclic Aromatic Hydrocarbons. J. Phys. Chem. A 1999, 103, 7487-7495.

${ }^{75}$ Khomenko, V. G.; Barsukov, V. Z.; Katashinskii, A. S. The 


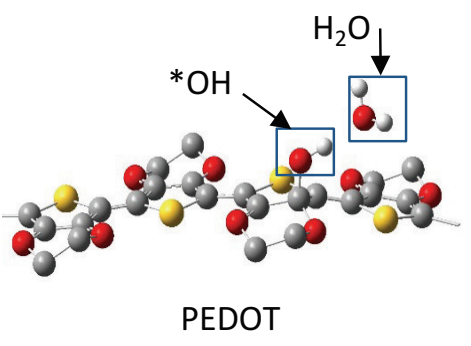

Polaron states

in PEDOT

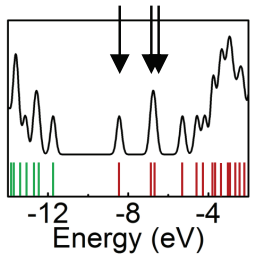

Catalytic Activity of Conducting Polymer towards Oxygen Reduction. Electrochim. Acta 2005, 50, 1675-1683.

FIG. 8: TOC 